\title{
Opinion formation models based on game theory
}

\author{
Alessandro Di Mare \\ Scuola Superiore di Catania \\ Catania, I-95123, Italy \\ E-mail: aldimare@ssc.unict.it \\ Vito Latora \\ Dipartimento di Fisica e Astronomia, Universitá di Catania, and \\ INFN, Sezione di Catania \\ Catania, I-95123, Italy \\ E-mail: latora@ct.infn.it \\ Received (received date) \\ Revised (revised date)
}

\begin{abstract}
A way to simulate the basic interactions between two individuals with different opinions, in the context of strategic game theory, is proposed. Various games are considered, which produce different kinds of opinion formation dynamics. First, by assuming that all individuals (players) are equals, we obtain the bounded confidence model of continuous opinion dynamics proposed by Deffuant et al. In such a model a tolerance threshold is defined, such that individuals with difference in opinion larger than the threshold can not interact. Then, we consider that the individuals have different inclinations to change opinion and different abilities in convincing the others. In this way, we obtain the socalled "Stubborn individuals and Orators" (SO) model, a generalization of the Deffuant et al. model, in which the threshold tolerance is different for every couple of individuals. We explore, by numerical simulations, the dynamics of the SO model, and we propose further generalizations that can be implemented.
\end{abstract}

Keywords: Sociophysics; opinion dynamics; game theory.

\section{Introduction}

The last years have seen an increasing interest in the physics community for the description and modeling of social systems. In particular, Monte Carlo simulations have become an important part of sociophysics ${ }^{1,2}$, enlarging the field of interdisciplinary applications of statistical physics. Most of the sociophysics models, such as that by Deffuant et al., that by Hegselmann-Krause, and the Sznajd model ${ }^{3,4}$, dealing with opinion dynamics and consensus formation, have the limit of considering that the individuals in a society are all equals. Conversely, an important feature of any real system, to be considered in the modeling of social system, is the presence of individuals with different inclinations to change idea ${ }^{6}$, as well as individuals with different abilities in convincing the others.

In this paper we focus on the model proposed by Deffuant et al. ${ }^{5}$, showing how the 
standard version of the model can be derived from basic principles in the framework of game theory. Moreover, in the context of game theory, the model can be easily generalized in different directions to take into account of the presence of individuals with different characteristics. In particular, we show how the introduction of a distribution of the individual inclinations to change and of the ability to convince the others, that produces what we have named the Stubborn Individuals and Orators (SO) model, can affect the opinion dynamics of a social group.

The paper is organized as follows. In Section 2 we briefly review the standard sociophysics models, in particular the model by Deffuant et al. In Section 3 we propose a way to derive models of opinion dynamics in the framework of game theory. The method we propose is based on a set of basic assumptions on the characteristics of the individuals (the players of the game) and on the payoffs for each of the possibile actions, and on the idea of Nash equilibrium for games with perfect information. We show how some simple models, including the model by Deffuant et al. can be derived. We then consider the SO model, a generalized model considering agents with different ability in convincing the others and with different inertia in changing ideas. In Section 4 we explore the dynamics of the SO model by means of extensive numerical simulations. In Section 5 we draw the conclusions and we outline further possible generalizations and future developments.

\section{Opinion formation models}

The standard models of opinions dynamics $2,3,4,7$ in sociophysics deal with $N$ individuals or agents. Each individual $i=1, \ldots, N$ is characterized, at time $t$, by an opinion $S_{i}(t)$. The opinions can be integer numbers (for instance +1 or -1 ) as in the Sznajd model ${ }^{8}$, or real numbers in the range $[0,1]$ as in the model by Deffuant et al. ${ }^{5}$ and in the Hegselmann and Krause model ${ }^{9}$. Each agent is in continuous interaction with the other agents. The opinion of an agent changes under the influence of the other individuals according to very simple deterministic rules. For instance, in the Sznajd model on a two-dimensional square lattice, at each time step, two randomly selected neighboring agents transfer their opinion to the six neighbors if and only if the two agents of the pair share the same opinion. In the model by Deffuant et al. ${ }^{5}$, at each time step $t$, two randomly selected neighboring agents $i$ and $j$ check their opinions $S_{i}(t)$ and $S_{j}(t)$ to see whether an exchange of opinion is possible. If the two opinions differ by more than a fixed threshold parameter $\epsilon(0<\epsilon<1)$, called the confidence bound, both opinions remain unchanged. If, instead, $\left|S_{i}(t)-S_{j}(t)\right|<\epsilon$, then each opinion moves in the direction of the other as:

$$
\left\{\begin{aligned}
S_{i}(t+1) & =S_{i}(t)+\mu\left[S_{j}(t)-S_{i}(t)\right] \\
S_{j}(t+1) & =S_{j}(t)-\mu\left[S_{j}(t)-S_{i}(t)\right]
\end{aligned}\right.
$$

with $\mu$ being a second tunable parameter $(0<\mu \leq 1 / 2)$. In the basic model, the threshold $\epsilon$ is taken as fixed in time and constant across the whole population. In particular, the value $\mu=1 / 2$ corresponds to the case in which the two opinions 
take their average $\left[S_{i}(t)-S_{j}(t)\right] / 2^{5}$.

To see if and how a consensus emerges out of initially different opinions, the models are usually started with a random initial distribution of opinions. The dynamics is followed until the system reaches an equilibrium state characterized by the existence of one or several opinion groups, according to the value of the control parameters of the models. For instance, the basic Sznajd model with random sequential updating always leads to a consensus on a regular lattice of any dimension $D$ (and even if more than two opinions are allowed). In particular, one observes a phase transition as a function of the initial concentration $p$ of up spins. If $p=0.5$, then, at the end of the dynamics, half of the samples will have $S_{i}=+1 \forall i$ and the remaining half will have $S_{i}=-1 \forall i$. For $p<1 / 2$ all samples end up with $S_{i}=-1 \forall i$, while for for $p>1 / 2$ they all end up in the other attractive fixed point $S_{i}=+1 \forall i{ }^{2}$. In the Deffuant model with $\mu=0.5$ instead, all opinions converge to a single central one for $\epsilon>1 / 2$, while for $\epsilon<1 / 2$ different opinions survive, with a number of surviving opinions that varies as $1 / \epsilon$, as also confirmed by analytical arguments ${ }^{11}$.

\section{Game theory and generalized models of opinion formation}

The main idea behind any of the previous models is to simulate how the opinions change in time by analyzing the very basic facts, that is: two individuals with different opinions on a given topic meet and discuss, trying to convince each other, or to find somehow a certain agreement about the topic. Of course, it is not obvious that the two individuals do find a common agreement on the topic, this depending basically on the specific characteristics of the two individuals (some of the individuals in a real social system are easy to convince, other are less flexible, some are good orators or distinguished for skills and power in convincing the others, while some others are timid and reserved), and also on some external factors (the time length and the strength of the interaction, the pressure of the external environment or of the dominant ideas and fashions).

In this paper we propose to examine the basic interactions between the two individuals within the framework of game theory ${ }^{10}$. For this reason, from now on, the two individuals will be also referred to as the two players. In particular, we make use of the concept of Nash equilibrium for games with perfect (or complete) information.

In general, a strategic game is a model of interacting decision makers. It consists of:

- a set of $N$ players or decision-makers;

- for each player $i(i=1, \ldots, N)$, a set of possible actions $A=\{a, b, c, \ldots\}$;

- for each player, preferences over the set of action profiles (i.e. the list of all the players' actions). 
One way to describe the player's preferences is to specify for each possible pair of actions, the action the player prefers, or to note that the player is indifferent between the actions. Alternatively one can represent the preferences by a payoff function, which associates a number with each action, in such a way that actions with higher numbers are preferred. More precisely, the payoff function $u$ represents a player's preferences if, for any couple of actions $a$ and $b$ in $A, u(a)>u(b)$ if and only if the player prefers $a$ to $b$. A simple example can be that of a person that is faced with three vacation packages, to New York, Paris and Venice. She prefers the package to Venice to the other two, which she regards as equivalent. Her preferences can be represented by any payoff function that assigns the same number to New York and Paris, and a higher number to Venice. For example, we can set $u(a)=u(b)=0$ and $u(c)=1$, where $a, b, c$ represent, respectively, the three packages. The fundamental hypothesis in game theory is that each player tries to maximize her benefit. This is usually called the hypothesis of rational choice, and means that, in any given situation, the decision-maker chooses the member of the available subset of $A$ that is best according to her preferences. Moreover, the strategic games considered here deal with situations in which actions are chosen once and for all (whereas there are games, named extensive games, allowing for the possibility that plans may be revised as they are carried out ${ }^{10}$ ). In the example above, the decision-maker will decide to go to Venice. In this simple example, we have only one decision-maker choosing an action from a set $A$, and caring only about this action. In the general case (that is of interest in this article), some of the variables that affect a player are the actions of other decision-makers, so that the decision-making problem is more challenging than that of an isolated player. The typical example is firms selling an item and competing for business. Each firm controls its price, but not the other firms's prices. Each firm cares however, about all the firms prices, because these prices affects its sales. How should a firm choose its prices in such a case ? In this case, the best action for any given player depends in general on the other players' actions. So when choosing an action, a player must have in mind the actions the other players will choose. That is, she must form a belief about the other players' actions. On what basis can such a belief be formed ? We consider here games in which each player's belief is derived from her past experience playing the game, and this experience is sufficiently extensive that she knows how her opponents will behave. No one tells her the actions the opponents will choose, but her previous involvement in the game leads her to be sure of these actions. These are called games with complete information, since in such games each player knows all the details of the game and of its elements.

In summary, in the strategic games we consider, there are two different components. First, each player chooses her action according to the model of rational choice, given her belief about the other players' actions. Second, every player's belief about the other players' actions is correct. These two components are embodied in the following definition of Nash equilibrium for such games ${ }^{10,12}$ : 
- A Nash equilibrium is an action profile $a^{*} \equiv\left(a_{1}^{*}, a_{2}^{*}, \ldots, a_{N}^{*}\right)$ - where $a_{1}^{*}$ denotes the action chosen by player $1, a_{2}^{*}$ the action chosen by player 2 and so on-, with the property that no player $i$ can do better by choosing an action different from $a_{i}^{*}$, given that every other player $j$ adheres to $a_{j}^{*}$.

This definition implies neither that a strategic game necessarily has a Nash equilibrium, nor that it has at most one. In general, some games have a single Nash equilibrium, some possess no Nash equilibrium and others have many Nash equilibria. A Nash equilibrium corresponds to a "steady state" of the system: if, whenever the game is played, the action profile is the Nash equilibrium $a^{*}$, then no player has a reason to choose any action different from her component of $a^{*}$. In practice, there is no pressure on the action profile to change. Expressed differently, a Nash equilibrium embodies a stable "social norm": if everyone else adheres to it, no individual wishes to deviate from it. The second component of the theory of Nash equilibrium (that the players' beliefs about each other's actions are correct) implies, in particular, that two players' beliefs about a third player's action are the same. For this reason, the condition is sometimes said to be that the players' "expectations are coordinated" 10.

The situations to which we wish to apply the theory of Nash equilibrium is the process of decision making in the formation of an opinion. We simulate the elementary interaction between individuals in a society by means of a strategic game. Then, we get a model of opinion formation, by iterating the game many times, i.e. by choosing at each time step a group of individuals and allowing them to play the game. In particular we assume that each game is played by only two players $(N=2)$, since here we limit to the particular case in which the dynamics of opinion formation is based on the continuous interaction between couples of individuals. (This is not always true. There are many real situations in which the elementary process of opinion formation is based on the mutual interaction of groups of more than two individuals. Nevertheless, our ideas can be generalized to games with $N>2$ ). We suggest a list of different possible games, the difference being in:

1. the number and kind of actions that one individual can choose from;

2. the characteristics of the two individuals.

For instance, in the simplest model (defined in Subsection 3.1), we assume that the individuals playing the game are all equals and can choose between two possibilities, either to mantain or to change their opinion. In the following, more complex models, we consider more than two actions for each player to choose from (e.g. introducing the possibility that the two players find an agreement). Moreover, we introduce a way to take into account that in a social group there are individual with different skills and abilities.

Before moving to the descriptions of the models and their equilibrium we want to stress that interactions in real social systems do not in general correspond exactly 
to the idealized setting described above (rational choice and complete information). For example, in some cases, the players do not have much experience with the game. In some other cases it could be useful to introduce non-rational players. Whether or not the notion of Nash equilibrium is appropriate in any given situation is a matter of judgment. In some cases, a poor fit with the idealized setting may be mitigated by other considerations. For example, inexperienced players may be able to draw conclusions about their opponents' likely actions from their experience in other situations, or from other sources. Ultimately, the test of the appropriateness of the notion of Nash equilibrium is whether it gives us insights into the problem at hand, that is to develop models of opinion formation.

\subsection{Game I}

In the most basic case, each of the two players of the game (named, from now on, player A and player B) can choose between two different actions: to mantain or to change opinion. As in any game we need to fix the actions' payoffs $u$. The payoff, for a player, is the function of her and the other player' actions. Of course, each player wants to convince the other one that her opinion is correct; on the other hand she does not want to accept easily the other player' opinion. Therefore, for each player, we fix the following payoffs:

- $\mathrm{u}=+\mathrm{a}$ if the other player changes her opinion

- $\mathrm{u}=+\mathrm{b}$ if the player keeps her opinion

- $\mathrm{u}=-\mathrm{b}$ if the other player keeps her opinion

- $\mathrm{u}=-\mathrm{a}$ if the player changes her opinion

where $a, b \in \Re$ and $0<b<a$. We take $b<a$, since we assume that a player gets the greatest satisfaction when is able to convince the opponent. Of course, this is just an hypothesis so that, in principle, also the choice $b>a$ would be an equally valid possibility. More in general, we should consider four different numbers for the payoffs, respectively: $a>0, b>0, c<0$ and $d<0$. Here, for the sake of simplicity, we assume $c=-b$ and $d=-a$. In table 1 , we report the payoff $u$ for players A and $\mathrm{B}$, for each of the strategies (action profiles). The two tables can be thought as two

Table 1. Game I: payoffs' tables for player A and player B

PAYOFFS FOR A

\begin{tabular}{lcc}
\hline & B changes & B keeps \\
\hline A changes & 0 & $-\mathrm{a}-\mathrm{b}$ \\
A keeps & $+\mathrm{a}+\mathrm{b}$ & 0 \\
\hline
\end{tabular}

PAYOFFS FOR B

\begin{tabular}{lcc}
\hline & B changes & B keeps \\
\hline A changes & 0 & $\mathrm{a}+\mathrm{b}$ \\
A keeps & $-\mathrm{a}-\mathrm{b}$ & 0 \\
\hline
\end{tabular}


matrices $M^{A}, M^{B}$, whose entry $m_{i j}$ represents the payoff, respectively for players $\mathrm{A}$ and $\mathrm{B}$, when $\mathrm{A}$ chooses the strategy $i$ and $\mathrm{B}$ chooses the strategy $j$. E.g., $m_{12}^{A}$ $\left(m_{12}^{B}\right)$ is the payoff for player $\mathrm{A}$ (player $\mathrm{B}$ ) when $\mathrm{A}$ chooses the action change and $\mathrm{B}$ chooses the action keep. Such a payoff is obtained by considering that player A is in the following condition: she changes her opinion, while the opponent mantains her opinion, therefore $m_{12}^{A}=-a-b$. On the other hand, player B mantains her opinion while the opponent changes her opinion, so that $m_{12}^{B}=+a+b$. With the same method we obtain the payoff for all the situations. The two matrices are:

$$
M^{A}=\left(\begin{array}{cc}
0 & -a-b \\
+a+b & 0
\end{array}\right) \quad M^{B}=\left(\begin{array}{cc}
0 & a+b \\
-a-b & 0
\end{array}\right)
$$

After creating the game, we want to foresee the actions that will be taken by the two players. It is easy to prove that the game has a single Nash equilibrium in $(2,2)$, i.e. when both players choose the strategy keep. In fact, if we fix that B chooses keep, then the player A can choose between a negative payoff $-a-b$ or 0 . Therefore she chooses the payoff 0, that corresponds to the strategy keep. In the same way, the player B chooses keep if we fix that A chooses keep. Therefore, neither player gets a greater payoff modifying her strategy, if the other player does not change her own. Consequently, two players of game I will always mantainin their own opinion, never reaching an agreement. In conclusion, a model of opinion dynamics, in which at each time step a couple of individuals is chosen at random among the $N$ individuals and play game I, will produce no time evolution: every individual will mantain the initial opinion.

\subsection{Game II}

We now introduce a new possibility: the agreement. This means that the two players can decide to change their own opinion with an intermediate one (not the initial opinions, nor the intermediate one needs to be better specified here). At this point we need to fix the payoff for the two new possible actions, considering that a player gets a certain satisfaction if she is able to shift the opponent's opinion to an intermediate one. We fix the following two new payoffs:

- $\mathrm{u}=+\mathrm{c}$ if the other player changes her opinion with an intermediate one

- $\mathrm{u}=$-c if the player changes her opinion with an intermediate one

where $c \in \Re$ and $c<a$. In table 2 we represent the payoffs for the various action profiles. The two matrices for the new conflicting opinion game are:

$$
M^{A}=\left(\begin{array}{ccc}
0 & -a-b & -a+c \\
+a+b & 0 & +b+c \\
+a-c & -b-c & 0
\end{array}\right) \quad M^{B}=\left(\begin{array}{ccc}
0 & a+b & a-c \\
-a-b & 0 & -b-c \\
-a+c & +b+c & 0
\end{array}\right)
$$

Notice that in this game, as in the previous one, the two matrices are trivially related since $M^{A}=-M^{B}$. The game has a single Nash equilibrium in the point $(2,2)$. 
Opinion formation models based on game theory.

Table 2. Game II: payoffs' tables for player A and player B

PAYOFFS FOR A

\begin{tabular}{lccc}
\hline & B changes & B keeps & B agrees \\
\hline A changes & 0 & $-\mathrm{a}-\mathrm{b}$ & $-\mathrm{a}+\mathrm{c}$ \\
A keeps & $+\mathrm{a}+\mathrm{b}$ & 0 & $+\mathrm{b}+\mathrm{c}$ \\
A agrees & $+\mathrm{a}-\mathrm{c}$ & $-\mathrm{b}-\mathrm{c}$ & 0 \\
\hline
\end{tabular}

PAYOFFS FOR B

\begin{tabular}{lccc}
\hline & B changes & B keeps & B agrees \\
\hline A changes & 0 & $+\mathrm{a}+\mathrm{b}$ & $+\mathrm{a}-\mathrm{c}$ \\
A keeps & $-\mathrm{a}-\mathrm{b}$ & 0 & $-\mathrm{b}-\mathrm{c}$ \\
A agrees & $-\mathrm{a}+\mathrm{c}$ & $+\mathrm{b}+\mathrm{c}$ & 0 \\
\hline
\end{tabular}

This means that, although the two players have, in principle, also the possibility of finding an agreement, they choose the strategy of mantaining their own ideas. Finally, the outcome of a model of opinion dynamics based on game II, would not produce results different from the trivial results of the model based on game I, i.e. no dynamics at all.

\subsection{Game III}

In the two previous games we have not taken into account the distances between the two players' opinions. Obviously, two individuals with close enough opinions can reach easier the agreement than two people with very different opinions. This has led to the introduction of the confidence bound mechanism in the model by Deffuant et al. (see Section 2). Hence, it can be useful also here, in the context of strategic games, to introduce a distance $d$ between the opinions of the players, and a corrective $d$-dependent term that makes the agreement easier in the game when $d$ is smaller. We fix the following payoffs:

- $\mathrm{u}=+\mathrm{a}$ if the other player changes her opinion

- $\mathrm{u}=+\mathrm{b}$ if the player keeps her opinion

- $\mathrm{u}=-\mathrm{b}$ if the other player keeps her opinion

- $\mathrm{u}=-\mathrm{a}$ if the player changes her opinion

- $\mathrm{u}=+\mathrm{c}+1 / \mathrm{d}$ if the other player changes her opinion with an intermediate one.

- $\mathrm{u}=-\mathrm{c}+1 / \mathrm{d}$ if the player changes her opinion with an intermediate one

where $d \in \Re$, and $d>0$. Notice that, if the two conditions $c+\frac{1}{d}>a$ and $-c+\frac{1}{d}>a$ are valid, then both players get a greater payoff by choosing the strategy to agree. The two conditions are both verified if $d<\frac{1}{a+c}$. The payoffs' matrices for the game are:

$$
M^{A}=\left(\begin{array}{ccc}
0 & -a-b & -a+c+\frac{1}{d} \\
+a+b & 0 & +b+c+\frac{1}{d} \\
+a-c+\frac{1}{d} & -b-c+\frac{1}{d} & \frac{2}{d}
\end{array}\right)
$$




$$
M^{B}=\left(\begin{array}{ccc}
0 & a+b & a-c+\frac{1}{d} \\
-a-b & 0 & -b-c+\frac{1}{d} \\
-a+c+\frac{1}{d} & +b+c+\frac{1}{d} & \frac{2}{d}
\end{array}\right)
$$

It is easy to prove that this game has two different equilibrium points. In fact:

when $d \leq \frac{1}{b+c}$, there is a Nash equilibrium in $(3,3)$.

when $d \geq \frac{1}{b+c}$, there is a Nash equilibrium in $(2,2)$.

If we define the confidence bound $\epsilon$ as the following function of the game parameters:

$$
\epsilon=\frac{1}{b+c}
$$

an opinion model based on $N$ individuals playing in randomly chosen couples game III, coincides exactly with the model by Deffuant et al. (with $\mu=0.5)^{5}$, discussed in Section 2. In fact, we can assume that the opinions are real numbers in the range $[0,1]$, as in the model by Deffuant et al., and we can start the $N$ individuals with a uniform random distribution of opinions. We then fix the three parameters $a, b, c$, that is equivalent to fixing a value of the confidence bound $\epsilon$ (a tuning of the parameters $a, b, c$ allows to have $\epsilon$ varying in the range $[0,1]$ ), while the distance $d$ depends on the two players' opinions and changes each time the game is played. Finally, the resulting model is the following. At each time step, two randomly chosen individuals play game III. When the distance $d$ between the two opinions is smaller than the confidence bound in Equation (6), then the two individual shift their opinions to the average one otherwise they keep their own opinions. This is nothing else than the model by Deffuant et al.

\subsection{Game IV: The SO model}

In the previous models, the individuals are considered all equals. They can have different opinions, but they have the same way to interact (i.e. in our framework, to play the game). Actually, this is far from being true in any real case. What makes the world interesting is the diversity of characters and behaviours we encounter. In particular, in a real social system, there are stubborn individual, i.e. individuals that do not change their own opinion easily, as well as people that change their opinion very easily. Moreover, there are orators, i.e. individuals with a certain influence in group processes and a well known ability in convincing the others ${ }^{13}$, as well as individuals that are not good in convincing the others. In order to take this into account in our models of opinion formation, we introduce two new variables, so that every individuals in our model is characterized by two values, the former representing the oratory ability and the latter representing the stubbornness of a person. We assume that each player is characterized by a couple of real numbers $p, q \in] 0,1[$ (the so-called characteristic parameters of the individual), where the first variable, $p$, represents the probability for a player to convince the opponent, and the second value, $q$, is the probability that a player keeps her own opinion. Considering, 
as in the previous cases, a game with only two players, $\mathrm{A}$ and $\mathrm{B}$, we assume that $p_{A}$, $q_{A}$ and $p_{B}, q_{B}$ are the characteristic parameters of the two players. Obviously, in the new game, the payoffs for each player should also depend on the four numbers $p_{A}, q_{A}, p_{B}, q_{B}$. This can be easily understood in the following way. Let us consider the action "A convinces B" and suppose to iterate the game $n$ times (with $n \ll 1$ ). In $n$ steps, the expected total payoff of $\mathrm{A}$ is $n a P$, where $a$ is the payoff for the same action in the previous game, and $P$ is the probability that A convinces $\mathrm{B}$. The latter is the product of $p_{A}$, the probability that $\mathrm{A}$ is able to convince, and $1-q_{B}$, that is the probability that B let himself to be convinced. Therefore, for every step A has a mean payoff equals to $a p_{A}\left(1-q_{B}\right)$. In a similar way we obtains the payoffs for all the other actions. Furthermore, we consider a distance-dependent term as in model III: since the average value of the payoffs is one fourth of that in the previous game, we choose $\frac{1}{4 d}$ as corrective term. Finally, the two matrices representing the payoffs for $\mathrm{A}$ and $\mathrm{B}$ are the following:

$$
\begin{gathered}
M^{A}=\left(\begin{array}{ccc}
a(P-X) & -a X-b Y & c P-a X+\frac{1}{4 d} \\
b Q+a P & b(Q-Y) & b Q+c P+\frac{1}{4 d} \\
a P-c X+\frac{1}{4 d} & -c X-b Y+\frac{1}{4 d} & c(P-X)+\frac{1}{2 d}
\end{array}\right) \\
M^{B}=\left(\begin{array}{ccc}
a(X-P) & a X+b Y & a X-c P+\frac{1}{4 d} \\
-b Q-a P & b(Y-Q) & -b Q-c P+\frac{1}{4 d} \\
c X-a P+\frac{1}{4 d} & c X+b Y+\frac{1}{4 d} & c(X-P)+\frac{1}{2 d}
\end{array}\right)
\end{gathered}
$$

where we have set $P=p_{A}\left(1-q_{B}\right), Q=q_{A}\left(1-p_{B}\right), X=p_{B}\left(1-q_{A}\right)$ and $Y=$ $q_{B}\left(1-p_{A}\right)$. The Nash equilibrium of the game changes as a function of the distance $d$ :

if $d \leq D\left(p_{B}, q_{A}\right)$ and $d \leq D\left(p_{A}, q_{B}\right)$, then there is a Nash equilibrium in $(3,3)$; if $d \geq D\left(p_{B}, q_{A}\right)$ and $d \geq D\left(p_{A}, q_{B}\right)$, then there is a Nash equilibrium in $(2,2)$; if $d \geq D\left(p_{B}, q_{A}\right)$ and $d \leq D\left(p_{A}, q_{B}\right)$, then there is a Nash equilibrium in $(2,3)$; if $d \leq D\left(p_{B}, q_{A}\right)$ and $d \geq D\left(p_{A}, q_{B}\right)$, then there is a Nash equilibrium in $(3,2)$; where we have defined the two following functions:

$$
\begin{aligned}
& D\left(p_{B}, q_{A}\right)=\frac{1}{4\left[b q_{A}+c p_{B}-(b+c) q_{A} p_{B}\right]} \\
& D\left(p_{A}, q_{B}\right)=\frac{1}{4\left[b q_{B}+c p_{A}-(b+c) p_{A} q_{B}\right]}
\end{aligned}
$$

The game we have proposed has three main differences with respect to the basic interaction mechanism in the model by Deffuant et al.:

1. It is possible that a player chooses the strategy "agreement" while the other 
player chooses the strategy "keep". This is not possible in the Deffuant et al. model.

2. The largest distance that allows for the agreement (confidence bound) depends on the characteristic parameters of the couple of players.

3. The shifting of the two players is in general different, so that it is necessary to introduce two different variables, $\mu$ and $\nu$ (one for each player), to indicate how much the two player shift their opinions.

If we indicate by $S_{A}(t)$ and $S_{B}(t)$ the two opinions at the time $t$, we can write the time evolution as:

$$
\left\{\begin{array} { c } 
{ S _ { A } ( t + 1 ) = S _ { A } ( t ) + \mu [ S _ { B } ( t ) - S _ { A } ( t ) ] } \\
{ S _ { j } ( t + 1 ) = S _ { B } ( t ) - \nu [ S _ { B } ( t ) - S _ { A } ( t ) ] }
\end{array} \quad \left\{\begin{array}{l}
0<\mu<0.5 \\
0<\nu<0.5
\end{array}\right.\right.
$$

where $\mu$ is a function of $p_{A}$ and $q_{B}$, while $\nu$ is a function of $p_{B}$ and $q_{A}$. To determine the functions $\mu\left(p_{A}, q_{B}\right)$ and $\nu\left(p_{B}, q_{A}\right)$ we notice that they should obey to the following obvious conditions:

$$
\left.\frac{\partial \mu}{\partial p_{A}}>0 ; \quad \frac{\partial \mu}{\partial q_{B}}<0 ; \quad \frac{\partial \nu}{\partial p_{B}}>0 ; \quad \frac{\partial \nu}{\partial q_{A}}<0 ; \quad \forall p_{B}, q_{B}, p_{A}, q_{A} \in\right] 0,1[
$$

For instance, the first two conditions state that $\mu\left(p_{A}, q_{B}\right)$ should be an increasing function of $p_{A}$ and a decreasing function of $q_{B}$. One possibility is to choose the two following functions:

$$
\mu\left(p_{A}, q_{B}\right)=\frac{p_{A}\left(1-q_{B}\right)}{2} \quad \nu\left(p_{B}, q_{A}\right)=\frac{p_{B}\left(1-q_{A}\right)}{2}
$$

Such a choice is in perfect agreement with the contraints $0<\mu<0.5$ and $0<$ $\nu<0.5$. Finally, we note that, if $d \leq D\left(p_{A}, q_{B}\right)$, then player B chooses the action "agreement" whatever is the strategy choosen by A. Analogously, if $d \leq D\left(p_{B}, q_{A}\right)$, then player A chooses to agree whatever is the strategy choosen by B. Therefore, the strategic game we have proposed can be resumed in the definition of the two following functions:

$$
\begin{aligned}
& \mu\left(p_{A}, q_{B}\right)=\left\{\begin{array}{cc}
\frac{p_{A}\left(1-q_{B}\right)}{2} & \text { if } d \leq D\left(p_{A}, q_{B}\right) \\
0 & \text { if } d>D\left(p_{A}, q_{B}\right)
\end{array}\right. \\
& \nu\left(p_{B}, q_{A}\right)=\left\{\begin{array}{cl}
\frac{p_{B}\left(1-q_{A}\right)}{2} & \text { if } d \leq D\left(p_{B}, q_{A}\right) \\
0 & \text { if } d>D\left(p_{B}, q_{A}\right)
\end{array}\right.
\end{aligned}
$$

In conclusion the "stubborn individuals and orators" ( $\mathrm{SO}$ ) model we propose, based on the strategic game IV, is the following. The model is fixed by choosing the values of the three control parameters $a, b, c$ (that is equivalent to choose a value of the confidence bound $\epsilon$ in the Deffuant et al model). The distance $d$ depends on the two players' opinions and changes each time the game is played. The $N$ individuals 
are, as usually, started with a random distribution of opinions in the range $[0,1]$. Moreover, each player $i(i=1,2, \ldots N)$ is now characterized by two variables, the characteristic parameters $p_{i}$ and $q_{i}$, distributed among the players according to two given distribution functions: $F_{1}(p)$ and $F_{2}(q)$. At each time step, two individuals, let say $\mathrm{A}$ and $\mathrm{B}$, are chosen at random and interact by playing the game. The results of the game depends on the distance $d$ between the two players' opinions and on the characteristic parameters $p_{A}, q_{A}, p_{B}, q_{B}$. The two players' opinions after the interaction (i.e. after the game) are shifted according to Equations (10), where the values of $\mu\left(p_{A}, q_{B}\right)$ and $\nu\left(p_{B}, q_{A}\right)$ are calculated through Equations (13) and (14), and through the functions in Equation (9). In particular, notice that, when $d>D\left(p_{A}, q_{B}\right), \mu$ is equal to zero and the opinion of player A remains unchanged: $S_{A}(t+1)=S_{A}(t)$. Analogously, when $d>D\left(p_{B}, q_{A}\right), \nu$ is equal to zero and $S_{B}(t+1)=S_{B}(t)$. Finally, the opinion dynamics model (the SO model) based on game IV, consists in iterating this procedure at each time steps.

\section{Montecarlo simulations of the SO model}

In this Section we turn our attention to the numerical simulation of the dynamics of the SO model. As shown in the previous section, the model depends on three different parameters $a, b$, and $c$. The three parameters are not independent. In fact $a$ does not appear explicitly in Equations (9), and plays the role of a normalization parameter, being only necessary to fix the maximum value of $b$ and $c$ (since we have assumed that $b<a$ and $c<a$. Consequently, the number of parameters can be reduced by fixing the value of $a$ and by considering the two normalized parameters:

$$
\beta=b / a \quad \gamma=c / a
$$

By definition, we have $0<\beta<1$ and $0<\gamma<1$. From Equations (12), we can notice that the mean value of $\mu$ and of $\nu$ is equal to $\langle\mu\rangle=\langle\nu\rangle=\frac{1}{8}$. Hence, if we suppose that the satisfaction of a player is a linear function of the shifting, then we can assume $\gamma=\frac{1}{8}$. In this way, the only independent parameter is $\beta$, since there are no reasons to fix a particular value for the satisfacion of a player to mantain the opinion.

Finally, in the numerical simulation we investigate the behaviour of the model for $\gamma=\frac{1}{8}$ and for different values of $\beta$. In particular, we consider a population of $N$ agents $(N>>1)$ with an initial (at time $t=0)$ heterogeneous distribution of opinions (every opinion in the range $[0,1]$ being equally probable). Each agent is described by two characteristic parameters $p$ and $q$. We assume that $p$ and $q$ are uniformly distributed in the range ]0,1[, i.e. that the two distribution functions $F_{1}(p)$ and $F_{2}(q)$ are equal to a constant. We evolve the system supposing that each player has the same probability to interact with any other (a more realistic possibility, not considered here, would be that of imagining the individuals interacting on a complex topology ${ }^{7}$. As explained in Section 3.4, at each time step, two randomly chosen agents try to convince each other of their opinions: they decide whether to 
change their opinion on the basis of the rules of game IV.

In Fig. 1, we show the number of large clusters, $N_{c}$, obtained at a fixed time $t_{f}$, as a function of $\beta$. A cluster is a group of people that share the same opinion. In the figure we consider only large clusters, i.e. clusters whose size is larger that $10 \%$ of the total population. We have considered $N=500$ and $t_{f}=5 \times 10^{5}$ time steps. Each of the points reported in the figure has been obtained as an average over 30 different realizations for the same value of $\beta$. We observe that $N_{c}$ is an

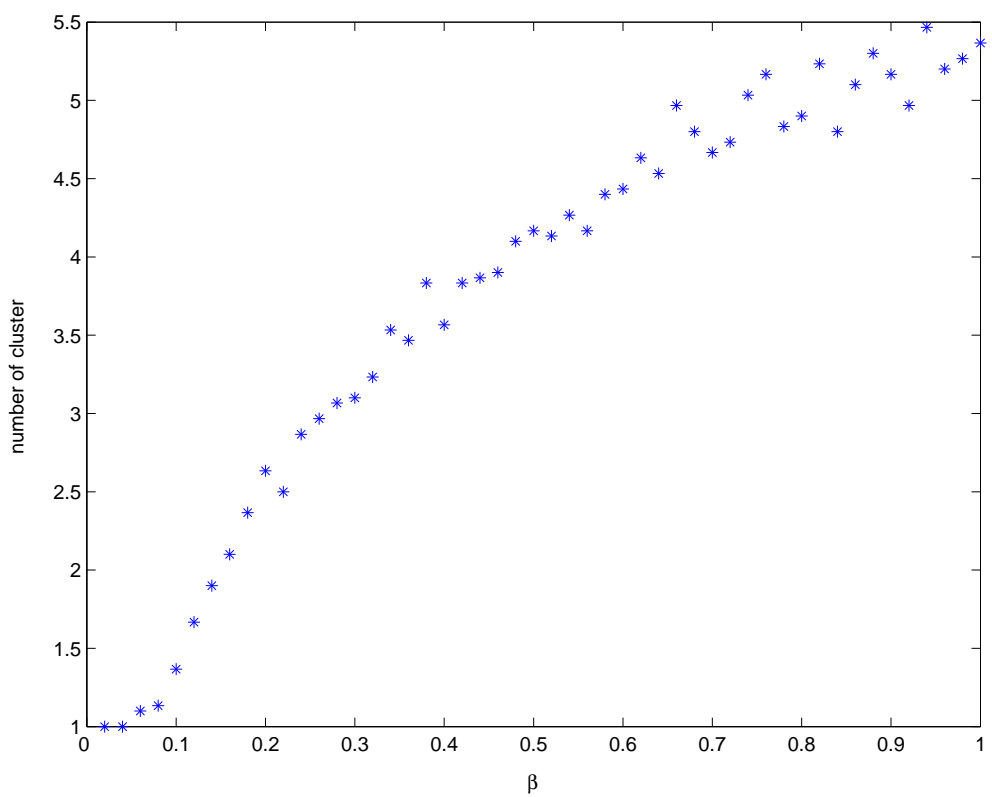

Fig. 1. SO model with $N=500, \gamma=1 / 8, \beta$ variable, and a uniform distribution of initial opinions and of the individual characteristic parameters $p$ and $q$. The number of clusters with a size larger than $0.1 N$ at time $t_{f}=5 \times 10^{5}$ is shown as a function of the parameter $\beta$.

increasing function of $\beta$. This is an obvious consequence of the model: in fact, $\beta$ represents the payoff (normalized to $a$ ) of a player if she mantains her opinion. Hence, an increasing value of $\beta$ causes in a player a natural inclination to find the agreement only with players with similar opinions, and therefore an increasing number of cluster. On the contrary, for small $\beta$, the players tend to change their opinions creating immediately a small number of clusters. As a further step, we have checked numerically whether the asymptotic state of the opinion distribution changes as a function of $\beta$. The simulations confirm the tendency of the system to reach a final equlibrium with a single large cluster of opinions for any value of $\beta$ in $[0,1]$. The time to reach the equilibrium depends strongly from $\beta$ and less from the different realizations of initial conditions. In the Fig. 2 we show the typical dynamical evolution for the case $\beta=0.8$. In each panel we plot the agents' opinions 
Opinion formation models based on game theory.

for six different times. For $t>5 \times 10^{6}$ we notice the presence of a few small groups
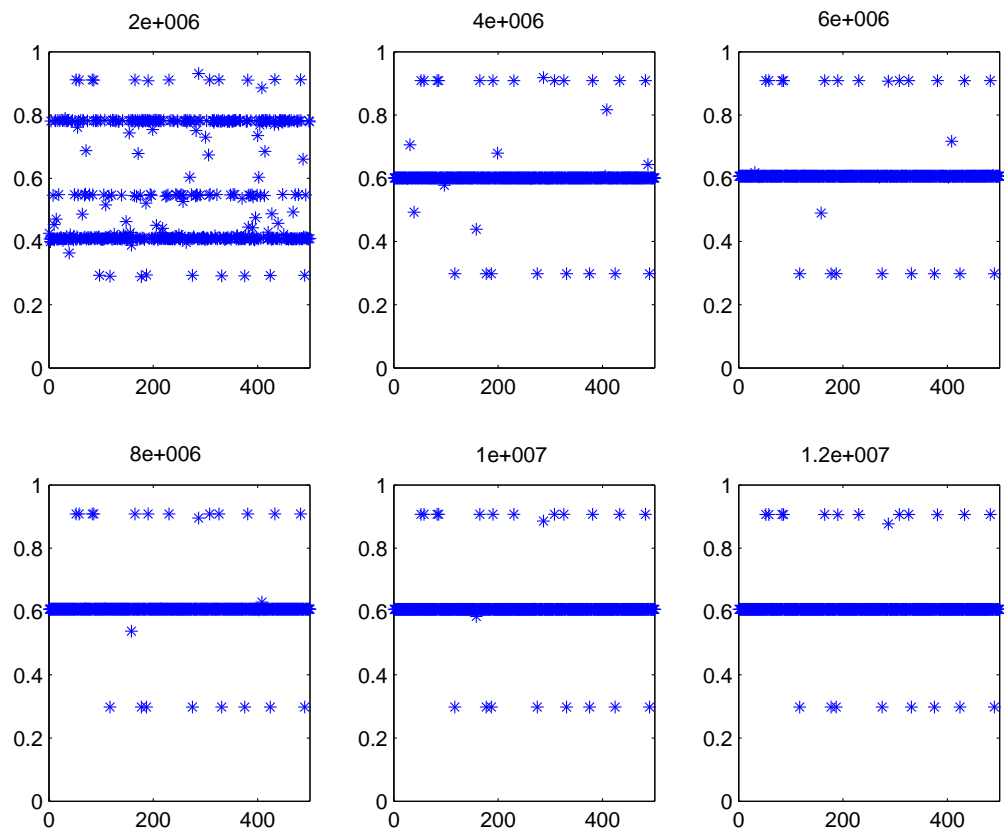

Fig. 2. SO model with $N=500, \gamma=1 / 8, \beta=0.8$, a uniform distribution of initial opinions and a uniform distribution of the individual characteristic parameters $p$ and $q$. We report the distribution of opinions among the 500 agents at six different times: $t_{1}=2 \times 10^{6}, t_{2}=4 \times 10^{6}$, $t_{3}=6 \times 10^{6}, t_{4}=8 \times 10^{6}, t_{5}=1 \times 10^{7}, t_{6}=1.2 \times 10^{7}$.

of opinions and a single large cluster containing about $96 \%$ of the total population, Similar results are obtained for other realizations of the initial conditions and for different values of $\beta$.

The presence of a single large cluster, observed at large times in the simulations, is a consequence of the all to all interactions (any couple of players is allowed to interact in this version of the model). If we analyze the first of equations (9), we notice that $D\left(p_{B}, q_{A}\right)$ takes only positive values because:

$$
b q_{A}+c p_{B}-(b+c) q_{A} p_{B}>0 \Leftrightarrow \frac{b}{p_{B}}+\frac{c}{q_{A}}>b+c
$$

is true $\forall p_{B}, q_{A} \in(0,1)$ Analogously, $D\left(p_{A}, q_{B}\right)$ is positive for any value of $p_{A}$ and $q_{B}$. This implies the existence, for any choice of the parameters, of a positive distance of opinions for which the agreemeent is possible. When we start the agents with a heterogeneous opinion distribution, for any given player $i$, it is certainly possible to find another player that can find an agreement with $i$. If we iterate the game IV for a long time, then there is a large probability that all the players reach a common agreement. Furthermore, if we consider an agent with an opinion next 
to 1 , she has a larger probability to play with another agent with a opinion lower than her. The same reasoning can be applied to an agent with an opinion close to 0 . Consequently, players with extreme opinions moves to central opinions and, after a large time we observe a single large cluster with a central opinion (e.g. at $S \approx 0.6$ in Fig. 2). An exception to this behaviour are those players who avoid the agreement with most of the other players. E.g. let us consider a player with $q=\frac{1}{2}$. It can be easily proven that such a player chooses the strategy to agree only when the distance with the opponent's opinion is very small. In fact, in the best case, i.e. when the opponent is an extremely good oratory characterized by $p=1$, a simple calculation gives $D(p, q)=0.08$. Consequently, the distance $d$ between the two individuals should be smaller than 0.08 for these two individuals to find a common agreement. If the dynamical evolution of opinions leads to isolate a player with $q=\frac{1}{2}$ at a distance larger that 0.08 from all the other players, such a player will never move from her opionion. This is the reason why, after a long time, we observe some (in general very few) isolated small clusters.

The tendency towards the formation of a single large cluster of opinions can be quantified, at each time step, by the calculation of the Gini coefficient of the opinion distribution. The Gini coefficient $G$ is a measure commonly used in economics and ecology to describe inequalities in the distribution of resource in a population ${ }^{14,15}$. In order to calculate the Gini coefficient of a generic empiric distribution one has first to computer the Lorenz curve of the distribution. In our case, the Lorenz curve of the opinion distribution is obtained in the following way. We divide the opinion range [0,1] in $M$ intervals (classes) of size $\Delta s$. Class $m$, with $m=1, \ldots M$, contains $n_{m}$ individuals, namely those having an opinion in the range $[(m-1) \Delta s, m \Delta s]$, with the normalization $\sum_{m=1}^{M} n_{m}=N$. The importance (richness) of a class is measured by the number of individuals it contains: the reachest class is the one containing the largest number of individuals. We then sort the classes in increasing order of $n_{m}$ (starting from the classes with the smallest number of individuals, up to the richest ones). Finally, in Fig. 3) we report (on the y-axis) the percentage of individuals, as a function of the percentage of the classes considered, in increasing order of importance (on the x-axis). This is the Lorenz curve of the opinion distribution. We name such a function $y=F(x)$. In particular, in Fig. 3) we consider the result of a simulation of the SO model with $N=10000$ individuals, and we report the Lorenz curves $F(x)$ obtained at four different times: $t_{1}=0, t_{2}=1.5 \times 10^{6}, t_{3}=3 \times 10^{6}$ and $t_{4}=4.5 \times 10^{6}$. Notice that, at time $t_{1}=0$, we have a uniform distribution of opinions and, as expected, the Lorenz curve $y=F(x)$ coincides with the line of perfect equality $y=x$. For larger times, the Lorenz curve tends more and more towards the Lorenz curve for the most heterogeneous distribution, that is $F(x)=0$ for $x \in[0,1[$, and $F(x)=1$ for $x=1$. This is eventually obtained when all the opinions are in the same class, so that the opinion classe are inequally populated: all of them are empty but a single one containing all the individuals. The Gini coefficient, $G$, can be calculated by comparing the Lorenz curve $y=F(x)$ of the distribution, with the line of perfect equality $y=x$ (the Lorenz curve of a perfectly 
Opinion formation models based on game theory.

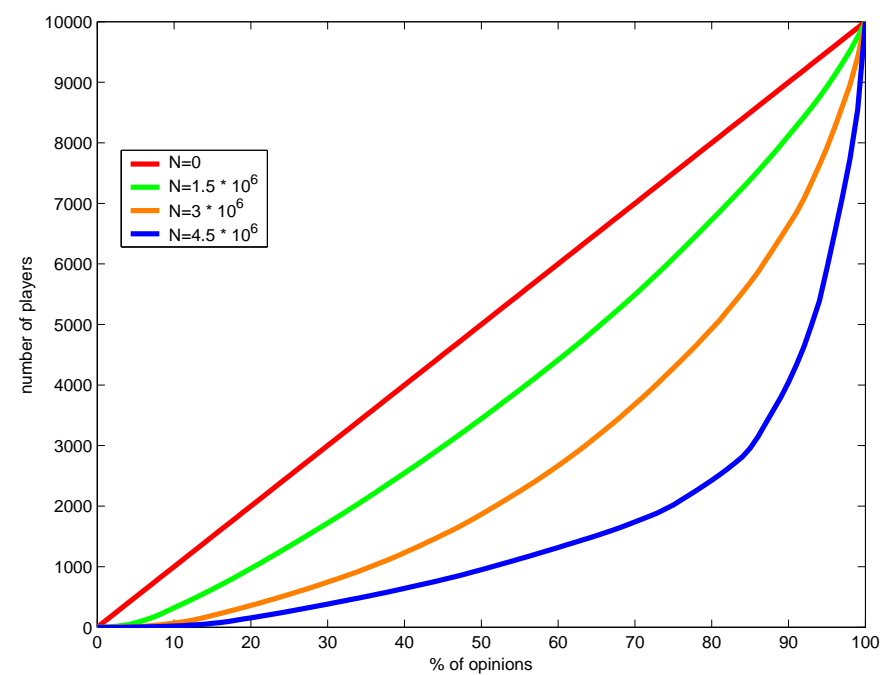

Fig. 3. SO model with $N=10000, \gamma=1 / 8, \beta=0.8$ and a uniform distribution of initial opinions and of the individual characteristic parameters $p$ and $q$. Lorenz curves of the opinion distribution. The number of players is reported, as a function of the percentage of opinions (see text for details), for four different times: $t_{1}=0, t_{2}=1.5 \times 10^{6}, t_{3}=3 \times 10^{6}$ and $t_{4}=4.5 \times 10^{6}$.

homogeneous distribution). $G$ is defined graphically as the ratio of two surfaces: the area between the line of perfect equality and the Lorenz curve, and the area between the line of perfect equality and the line of perfect inequality. One gets:

$$
G=1-2 \int_{0}^{1} F(x) d x
$$

By definition, the coefficient $G$ ranges from a minimum value of zero, in the case in which all the classes are equivalently occupied, to a maximum value of 1 in a population in which every class except one has a size of zero, i.e. all the individuals share the same opinion. In Fig. 4 we report the Gini coefficient as a function of time for three different dynamical evolutions of the SO model, respectively corresponding to the cases $\beta=0.02, \beta=0.4$ and $\beta=1$. These three cases are a good sample of the model behaviours, since by definition the parameter $\beta$ is allowed to vary in the range $[0,1]$. For each value of $\beta$ considered, the system evolves towards a state with a high Gini coefficient. We notice that:

1. The qualitative dynamical behaviour does not change with $\beta$. The system reaches an asymptotic value of $G$ which is very close to 1 . This denotes the presence of a single large cluster (and possibly few small clusters), for any value of $\beta$. In practice, extreme heterogeneity is not allowed in the SO model.

2. The values of $\beta$ influence the dynamics in two ways: A) the stationary value 


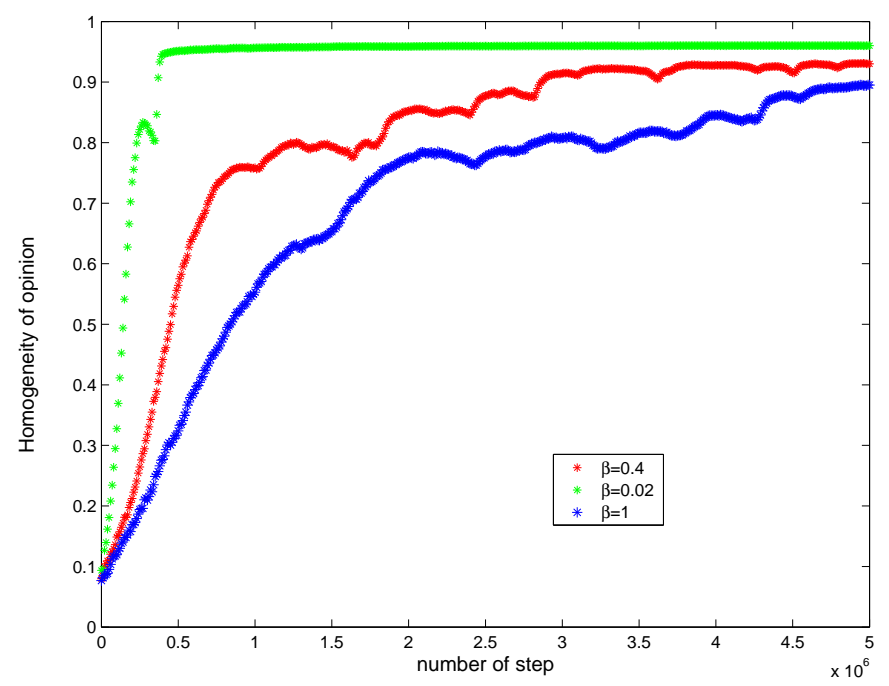

Fig. 4. SO model with $N=10000, \gamma=1 / 8$, a uniform distribution of initial opinions and a uniform distribution of the individual characteristic parameters $p$ and $q$. The Gini coefficient $G$ is reported as a function of time. The three curves correspond to three different values of $\beta$, namely, from bottom to top, $\beta=1,0.4$, and 0.02 .

of $G$ is smaller for higher values of $\beta$; B) the converge dynamics is slower for higher values of $\beta$.

The existence of a single large cluster in the numerical simulations of the SO model is largely due to the fact that we have assumed a uniform distribution of the individual characteristic parameters $p$ and $q$. Other distributions of the parameters, as for instance $F_{1}(p)$ and $F_{2}(q)$ gaussian distributed, are equally interesting to be investigated. In particualar we expect that the model can give different results by tuning mean value and standard deviation of the gaussian distribution of characteristic parameters.

\section{Conclusions}

In this paper we have shown how strategic game theory can find useful in the modeling of opinion formations, as a way to simulate the basic interaction mechanisms between two individuals. In particular, we have shown how various models of opinion formation can be obtained by just changing the rules of the game, i.e. the number and the kind of actions an individual can choose from, and also the very same characteristics of the individuals. In the context of one of the simplest game considered we were able to derive, by basic principles, a well known model of opinion dynamics, such as the Deffuant et al. model. Then, we have generalized the Deffuant et al. model by introducing in the game social individuals with two characteristic parameters, respectively representing different inclinations to change 
opinion and different abilities in convincing the others. Such a game produce the so called Stubborn Individuals and Orators (SO) model. We have investigated numerically the dynamics of the SO model in the case of all-to-all interactions, and in the simplest possible case of a uniform distribution of characteristic parameters. In such a case the model converges to a single dominant opinion for any value of the control parameters. This model can still be generalized by using different distributions of characteristic parameters, or by allowing the individuals to interact only with the neigbours in a network. Many other models can be introduced in the context of strategic game theory, so that we hope that our paper can stimulate further research in the field of sociophysics.

\section{References}

1. W. Weidlich, Sociodynamics; A Systematic Approach to Mathematical Modelling in the Social Sciences. Harwood Academic Publishers, 2000.

2. D. Stauffer, The Monte Carlo Method in the Physical Sciences, edited by J. E. Gubernatis, AIP Conf. Proc. 690, 147 (2003).

3. D.Stauffer, Sociophysics Simulations, Computer in Science and Engineering (2003 IEEE)

4. S. Fortunato, D. Stauffer, cond-mat/0501730 in press.

5. G. Deffuant, D. Neau, F. Amblard and G. Weisbuch, Adv. Complex Syst. 3, 87 (2000). G. Deffuant, F. Amblard, and J.-P. Nadal, Complexity 7, 2002; G. Deffuant, F. Amblard, G. Weisbuch and T. Faure, Journal of Artificial Societies and Social Simulations 5, issue 4, paper 1 (jasss.soc.surrey.ac.uk) (2002). G. Weisbuch Eur. Phys. J. B 38, 339 (2004).

6. A. Pluchino, V. Latora, and A. Rapisarda, Int. J. Mod. Phys. C 16, 515 (2005).

7. S. Boccaletti, V. Latora. Y. Moreno, M. Chavez and D.-U. Hwang, Phys. Rep. 424, 175 (2006).

8. K. Sznajd-Weron and J. Sznajd, Int. J. Mod. Phys. C 11, 1157 (2000).

9. R. Hegselmann and U. Krause, Journal of Artificial Societies and Social Simulation 5, issue 3, paper 2 (jasss.soc.surrey.ac.uk) (2002).

10. M. J. Osborne, An Introduction to Game Theory, (Oxford University Press, 2002).

11. E. Ben-Naim, P.L. Krapivsky and S. Redner, Physica D 183, 190 (2003).

12. J.F. Nash, Proc. Natl. Acad. Sci. U.S.A. 36, 48 (1950).

13. V. Latora and M. Marchiori, Preprint cond-mat/0402050.

14. C. Dagum, Écon. Appl. 33, 327 (1980).

15. M.O.Lorenz, Publications of the American Statistical Association 9, 209 (1905). 